\title{
Extensions of Toric Varieties
}

\author{
Mesut Şahin* \\ Department of Mathematics \\ Çankırı Karatekin University, 18100, Çankırı, Turkey \\ mesutsahin@karatekin.edu.tr
}

Submitted: Dec 24, 2010; Accepted: Apr 11, 2011; Published: Apr 21, 2011

Mathematics Subject Classifications: 14M25, 13D40,14M10,13D02

\begin{abstract}
In this paper, we introduce the notion of "extension" of a toric variety and study its fundamental properties. This gives rise to infinitely many toric varieties with a special property, such as being set theoretic complete intersection or arithmetically Cohen-Macaulay (Gorenstein) and having a Cohen-Macaulay tangent cone or a local ring with non-decreasing Hilbert function, from just one single example with the same property, verifying Rossi's conjecture for larger classes and extending some results appeared in literature.
\end{abstract}

\section{Introduction}

Toric varieties are rational algebraic varieties with special combinatorial structures making them objects on the crossroads of different areas such as algebraic statistics, dynamical systems, hypergeometric differential equations, integer programming, commutative algebra and algebraic geometry.

Affine extensions of a toric curve has been introduced for the first time by Arslan and Mete [2] inspired by Morales' work [10] and used to study Rossi's conjecture saying that Gorenstein local rings has non-decreasing Hilbert functions. Later, we have studied settheoretic complete intersection problem for projective extensions motivated by the fact that every projective toric curve is an extension of another lying in one less dimensional projective space [17]. Our purpose here is to emphasize the nice behavior of toric varieties (of any dimension this time) under the operation of extensions and we hope that this

${ }^{*}$ I would like to thank M. Barile, M. Morales, M.E. Rossi and A. Thoma for their invaluable comments on the preliminary version of the present paper. A part of this paper was written while I was visiting the Abdus Salam International Centre for Theoretical Physics (ICTP), Trieste, Italy. I acknowledge the support and hospitality. I also would like to thank the anonymous referee for very helpful suggestions improving the presentation of the paper.

The ELECTROnic Journal of COMbinatorics 18 (2011), \#P93 
approach will provide a rich source of classes for studying many other conjectures and open problems.

In the first part of the present paper we note that affine extensions can be obtained by gluing semigroups and thus their minimal generating sets can be obtained by adding a binomial, see Proposition 2.4. In the projective case a similar result holds under a mild condition, see Proposition 2.7, which is not true in general by Example 2.6 since projective extensions are not always obtained by gluing. In particular, if we start with a set theoretic complete intersection, arithmetically Cohen-Macaulay or Gorenstein toric variety, then we obtain infinitely many toric varieties having the same property, generalizing [19].

We devote the second part for the local study of extensions of toric varieties. Namely, if a toric variety has a Cohen-Macaulay tangent cone or at least its local ring has a nondecreasing Hilbert function, then we prove that its nice extensions share these properties supporting Rossi's conjecture for higher dimensional Gorenstein local rings and extending results appeared in [1, Proposition 4.1] and [2, Theorem 3.6]. Similarly, we show that if its local ring is of homogeneous type, then so are the local rings of its extensions. Local properties of toric varieties of higher dimensions have not been studied extensively, although there is a vast literature about toric curves, see $[12,16],[3,18]$ and references therein. This paper might be considered as a first modest step towards the higher dimensional case.

\section{Prelimineries}

Throughout the paper, $K$ is an algebraically closed field of any characteristic. Let $S$ be a subsemigroup of $\mathbb{N}^{d}$ generated by $\mathbf{m}_{1}, \ldots, \mathbf{m}_{n}$. If we set $\operatorname{deg}_{S}\left(x_{i}\right)=\mathbf{m}_{i}$, then $S$-degree of a monomial is defined by

$$
\operatorname{deg}_{S}\left(\mathbf{x}^{\mathbf{b}}\right)=\operatorname{deg}_{S}\left(x_{1}^{b_{1}} \cdots x_{n}^{b_{n}}\right)=b_{1} \mathbf{m}_{1}+\cdots+b_{n} \mathbf{m}_{n} \in S .
$$

The toric ideal of $S$, denoted $I_{S}$, is the prime ideal in $K\left[x_{1}, \ldots, x_{n}\right]$ generated by the binomials $\mathbf{x}^{\mathbf{a}}-\mathbf{x}^{\mathbf{b}}$ with $\operatorname{deg}_{S}\left(\mathbf{x}^{\mathbf{a}}\right)=\operatorname{deg}_{S}\left(\mathbf{x}^{\mathbf{b}}\right)$. The set of zeroes in $\mathbb{A}^{n}$ is called the toric variety of $S$ and is denoted by $V_{S}$. The projective closure of a variety $V$ will be denoted by $\bar{V}$ as usual and we write $\bar{S}$ for the semigroup defining the toric variety $\bar{V}_{S}$.

Denote by $S_{\ell, \mathbf{m}}$ the affine semigroup generated by $\ell \mathbf{m}_{1}, \ldots, \ell \mathbf{m}_{n}$ and $\mathbf{m}$, where $\ell$ is a positive integer. When $\mathbf{m} \in S$, we define $\delta(\mathbf{m})$ (respectively $\Delta(\mathbf{m})$ ) to be the minimum (respectively maximum) of all the sums $s_{1}+\cdots+s_{n}$ where $s_{1}, \ldots, s_{n}$ are some non-negative integers such that $\mathbf{m}=s_{1} \mathbf{m}_{1}+\cdots+s_{n} \mathbf{m}_{n}$.

Definition 2.1 (Extensions). With the preceding notation, we say that the affine toric variety $V_{S_{\ell, \mathbf{m}}} \subset \mathbb{A}^{n+1}$ is an extension of $V_{S} \subset \mathbb{A}^{n}$, if $\mathbf{m} \in S$, and $\ell$ is a positive integer relatively prime to a component of $\mathbf{m}$. A projective variety $\bar{E} \subset \mathbb{P}^{n+1}$ will be called an extension of another one $\bar{X} \subset \mathbb{P}^{n}$ if its affine part $E$ is an extension of the affine part $X$ of $\bar{X}$.

Remark 2.2. 1. Notice that $\overline{V_{S}}=V_{\bar{S}}, I_{S} \subset I_{S_{\ell, \mathbf{m}}}$ and $I_{\bar{S}} \subset I_{\bar{S}_{\ell, \mathbf{m}}}$. 
2. The question of whether or not $I_{S_{\ell, \mathbf{m}}}$ (resp. $I_{\bar{S}_{\ell, \mathbf{m}}}$ ) has a minimal generating set containing a minimal generating set of $I_{S}\left(\operatorname{resp} . I_{\bar{S}}\right)$ is not trivial.

3. This definition generalizes the one given for monomial curves in $[2,17]$.

4. In [19], special extensions for which $\ell$ equals to a multiple of $\delta(\mathbf{m})$ has been studied without referring to them as extensions.

Now we recall the definition of gluing semigroups introduced first by Rosales [14] and used by different authors to produce certain family of examples in different context, see for example $[3,7,11]$. Let $T=T_{1} \bigsqcup T_{2}$ be a decomposition of a set $T \subset \mathbb{N}^{d}$ into two disjoint proper subsets. The semigroup $\mathbb{N} T$ is called a gluing of $\mathbb{N} T_{1}$ and $\mathbb{N} T_{2}$ if there exists a nonzero $\alpha \in \mathbb{N} T_{1} \bigcap \mathbb{N} T_{2}$ such that $\mathbb{Z} \alpha=\mathbb{Z} T_{1} \cap \mathbb{Z} T_{2}$.

Remark 2.3. If $S$ is a gluing of $S_{1}$ and $S_{2}$ then $I_{S}=I_{S_{1}}+I_{S_{2}}+\left\langle F_{\alpha}\right\rangle$, where $F_{\alpha}=$ $x_{1}^{b_{1}} \cdots x_{n}^{b_{n}}-y_{1}^{c_{1}} \cdots y_{n}^{c_{n}}$ with $\operatorname{deg}_{S}\left(F_{\alpha}\right)=\operatorname{deg}_{S}\left(x_{1}^{b_{1}} \cdots x_{n}^{b_{n}}\right)=\operatorname{deg}_{S}\left(y_{1}^{c_{1}} \cdots y_{n}^{c_{n}}\right)=\alpha$. Since $F_{\alpha}$ is a non-zero divisor, the minimal free resolution of $I_{S}$ can be obtained by tensoring out the given minimal free resolutions of $I_{S_{1}}$ and $I_{S_{2}}$, and then applying the mapping cone construction. It is also standard to deduce that the coordinate ring of $V_{S}$ is CohenMacaulay (Gorenstein) when the coordinate rings of $V_{S_{1}}$ and $V_{S_{2}}$ are so. The converse is false as there are Cohen-Macaulay (Gorenstein) toric curves in $\mathbb{A}^{4}$ which can not be obtained by gluing two toric curves.

The first observation is that affine extensions can be obtained by gluing.

Proposition 2.4. If the toric variety $V_{S_{\ell, \mathbf{m}}} \subset \mathbb{A}^{n+1}$ is an extension of $V_{S} \subset \mathbb{A}^{n}$, then $S_{\ell, \mathbf{m}}$ is the gluing of $\mathbb{N} T_{1}$ and $\mathbb{N} T_{2}$, where $T_{1}=\left\{\ell \mathbf{m}_{1}, \ldots, \ell \mathbf{m}_{n}\right\}$ and $T_{2}=\{\mathbf{m}\}$. Consequently, $I_{S_{\ell, \mathbf{m}}}=I_{S}+\langle F\rangle$, where $F=x_{n+1}^{\ell}-x_{1}^{s_{1}} \cdots x_{n}^{s_{n}}$.

Proof. First of all, $S=\mathbb{N}\left\{\mathbf{m}_{1}, \ldots, \mathbf{m}_{n}\right\}, S_{\ell, \mathbf{m}}=\mathbb{N} T$, where the set $T=T_{1} \sqcup T_{2}, T_{1}=$ $\left\{\ell \mathbf{m}_{1}, \ldots, \ell \mathbf{m}_{n}\right\}$ and $T_{2}=\{\mathbf{m}\}$. We claim that $S_{\ell, \mathbf{m}}$ is the gluing of its subsemigroups $\mathbb{N} T_{1}$ and $\mathbb{N} T_{2}$. To this end we show that $\mathbb{Z} T_{1} \cap \mathbb{Z} T_{2}=\mathbb{Z} \alpha$, where $\alpha=\ell \mathbf{m} \in \mathbb{N} T_{1} \cap \mathbb{N} T_{2}$.

Since $\ell \mathbf{m}=s_{1} \ell \mathbf{m}_{1}+\cdots+s_{n} \ell \mathbf{m}_{n}$ with non-negative integers $s_{i}$, we have clearly $\mathbb{Z} T_{1} \cap$ $\mathbb{Z} T_{2} \supseteq \mathbb{Z} \alpha$. Take $z \mathbf{m}=z_{1} \ell \mathbf{m}_{1}+\cdots+z_{n} \ell \mathbf{m}_{n} \in \mathbb{Z} T_{1} \cap \mathbb{Z} T_{2}$ and note that $z \mathbf{m}=\ell\left(z_{1} \mathbf{m}_{1}+\right.$ $\left.\cdots+z_{n} \mathbf{m}_{n}\right)$. Since $\ell$ is relatively prime to a component of $\mathbf{m}$ by assumption, it follows that $\ell$ divides $z$ and thus $z \mathbf{m} \in \mathbb{Z} \alpha$ yielding $\mathbb{Z} T_{1} \cap \mathbb{Z} T_{2} \subseteq \mathbb{Z} \alpha$. By the relation between the corresponding ideals, we have $I_{S_{\ell, \mathbf{m}}}=I_{S}+\langle F\rangle$, since $I_{T_{1}}=I_{S}$ and $I_{T_{2}}=0$.

Since $F=x_{n+1}^{\ell}-x_{1}^{s_{1}} \cdots x_{n}^{s_{n}}$ is a non-zero divisor of $R\left[x_{n+1}\right] / I_{S} R\left[x_{n+1}\right]$, where $R=$ $K\left[x_{1}, \ldots, x_{n}\right]$, and $\sqrt{I_{S_{\ell, \mathbf{m}}}}=\sqrt{\sqrt{I_{S}}+\sqrt{F}}$ the following is immediate.

Corollary 2.5. If $V_{S} \subset \mathbb{A}^{n}$ is a set theoretic complete intersection, arithmetically CohenMacaulay (Gorenstein), so are its extensions $V_{S_{\ell, \mathbf{m}}} \subset \mathbb{A}^{n+1}$. 


\subsection{Projective Extensions}

Since projective extensions can not be obtained by gluing in general, see [17], we study them separately in this section. Contrary to the case of affine extensions, it is not true in general that a minimal generating set of a projective extension of $V_{\bar{S}}$ contains a minimal generating set of $I_{\bar{S}}$ as illustrated by the following example.

Example 2.6. If $S=\mathbb{N}\{1,4,5\}$, then the projective monomial curve $V_{\bar{S}}$ in $\mathbb{P}^{3}$ is defined by $\bar{S}=\mathbb{N}\{(5,0),(4,1),(1,4),(0,5)\}$. Consider the projective extension $V_{\bar{S}_{1,10}}$ defined by the semigroup

$$
\bar{S}_{1,10}=\mathbb{N}\{(10,0),(9,1),(6,4),(5,5),(0,10)\} .
$$

It is easy to see (use e.g. Macaulay [4]) that the set $\left\{F_{1}, F_{2}, F_{3}, F_{4}, F_{5}\right\}$ constitutes a reduced Gröbner basis (and a minimal generating set) for the ideal $I_{\bar{S}}$ with respect to the reverse lexicographic order with $x_{1}>x_{2}>x_{3}>x_{0}$, where

$$
\begin{aligned}
& F_{1}=x_{1}^{4}-x_{0}^{3} x_{2} \\
& F_{2}=x_{2}^{4}-x_{1} x_{3}^{3} \\
& F_{3}=x_{1}^{2} x_{3}^{2}-x_{0} x_{2}^{3} \\
& F_{4}=x_{1}^{3} x_{3}-x_{0}^{2} x_{2}^{2} \\
& F_{5}=x_{1} x_{2}-x_{0} x_{3} .
\end{aligned}
$$

A computation shows that the set $\left\{F_{1}, F_{4}, F_{5}, F, F_{6}, F_{7}\right\}$ is a reduced Gröbner basis for $I_{\bar{S}_{1,10}}$ with respect to the reverse lexicographic order with $x_{1}>x_{2}>x_{3}>x_{4}>x_{0}$, where

$$
\begin{aligned}
F & =x_{3}^{2}-x_{0} x_{4} \\
F_{6} & =x_{2}^{3}-x_{1}^{2} x_{4} \\
F_{7} & =x_{1}^{3} x_{4}-x_{0} x_{2}^{2} x_{3}
\end{aligned}
$$

We observe now that $F_{7}=x_{2}^{2} F_{5}-x_{1} F_{6}$ and that the set $\left\{F_{1}, F_{4}, F_{5}, F, F_{6}\right\}$ is a minimal generating set of $I_{\bar{S}_{1,10}}$. The fact that no minimal generating set of $I_{\bar{S}}$ extends to a minimal generating set of $I_{\bar{S}_{1,10}}$ follows from the observation that $\mu\left(I_{\bar{S}}\right)=\mu\left(I_{\bar{S}_{1,10}}\right)(=5)$, where $\mu(\cdot)$ denotes the minimal number of generators.

Notice that the previous example reveals why minimal generating sets need not extend when $\ell<\delta(\mathbf{m})$. Next, we show that this can be avoided as long as $\ell \geq \delta(\mathbf{m})$. So, we compute a Gröbner basis for $I_{\bar{S}_{\ell, \mathbf{m}}}$ using the Proposition 2.4 and the fact that if $\mathcal{G}$ is a Gröbner basis for the ideal of an affine variety with respect to a term order refining the order by degree, then the homogenization of $\mathcal{G}$ is a Gröbner basis for the ideal of its projective closure.

Proposition 2.7. If $\mathcal{G}$ is a reduced Gröbner basis for $I_{\bar{S}}$ with respect to a term order $\succ$ making $x_{0}$ the smallest variable and $\ell \geq \delta(\mathbf{m})$, then $\mathcal{G} \cup\{F\}$ is a reduced Gröbner basis for $I_{\bar{S}_{\ell, \mathrm{m}}}$ with respect to a term order refining $\succ$ and making $x_{n+1}$ the biggest variable and thus $I_{\bar{S}_{\ell, \mathbf{m}}}=I_{\bar{S}}+\langle F\rangle$, where $F=x_{n+1}^{\ell}-x_{0}^{\ell-\delta(\mathbf{m})} x_{1}^{s_{1}} \cdots x_{n}^{s_{n}}$. 
Proof. Let $\mathcal{G}=\left\{F_{1}, \ldots, F_{k}\right\}$. If we dehomogenize the polynomials in $\mathcal{G}$ by substituting $x_{0}=1$, we get a reduced Gröbner basis $\left\{G_{1}, \ldots, G_{k}\right\}$ for $I_{S}$ with respect to $\succ$ which refines the order by degree. From Proposition 2.4, we know that $I_{S_{\ell, \mathbf{m}}}=$ $I_{S}+\langle G\rangle=\left\langle G_{1}, \ldots, G_{k}, G\right\rangle$, where $G=F\left(1, x_{1}, \ldots, x_{n}\right)$. Since $\operatorname{LM}\left(G_{i}\right) \in K\left[x_{1}, \ldots, x_{n}\right]$ and $\operatorname{LM}(G)=x_{n+1}^{\ell}$, it follows that $\operatorname{gcd}\left(\operatorname{LM}\left(G_{i}\right), \operatorname{LM}(G)\right)=1$, for all $i$. This implies that the set $\left\{G_{1}, \ldots, G_{k}, G\right\}$ is a Gröbner basis for $I_{S_{\ell, \mathbf{m}}}$ with respect to a term order refining the order by degree and $\succ$. Hence, their homogenizations constitute the required Gröbner basis for $I_{\bar{S}_{\ell, \mathbf{m}}}$ as claimed.

Now, if $\operatorname{LM}\left(F_{i}\right)$ does not divide $\operatorname{NLM}(F):=x_{0}^{\ell-\delta(\mathbf{m})} x_{1}^{s_{1}} \cdots x_{n}^{s_{n}}$, it follows that $\mathcal{G} \cup\{F\}$ is reduced as $\mathcal{G}$ is also. Otherwise, i.e., $\operatorname{NLM}(F)=\operatorname{LM}\left(F_{i}\right) x_{0}^{\ell-\delta(\mathbf{m})} M$, for some monomial $M$ in $K\left[x_{1}, \ldots, x_{n}\right]$, we replace $\operatorname{NLM}(F)$ by $T_{i} x_{0}^{\ell-\delta(\mathbf{m})} M$, since $\operatorname{deg}_{S}\left(\operatorname{LM}\left(F_{i}\right)\right)=\operatorname{deg}_{S}\left(T_{i}\right)$, which means that the new binomial $F=x_{n+1}^{\ell}-T_{i} x_{0}^{\ell-\delta(\mathbf{m})} M \in I_{\bar{S}_{\ell, \mathbf{m}}}$. Since $\mathcal{G}$ is reduced and $F_{i}$ are irreducible binomials, no $L M\left(F_{j}\right)$ divides $T_{i} x_{0}^{\ell-\delta(\mathbf{m})} M$. Therefore, the set $\mathcal{G} \cup\{F\}$ is reduced as desired. Thus, we obtain $I_{\bar{S}_{\ell, \mathbf{m}}}=I_{\bar{S}}+\langle F\rangle$.

As in the affine case we have the following.

Corollary 2.8. If $V_{\bar{S}} \subset \mathbb{P}^{n}$ is a set theoretic complete intersection, arithmetically CohenMacaulay (Gorenstein), so are its extensions $V_{\bar{S}_{\ell, \mathbf{m}}} \subset \mathbb{P}^{n+1}$ provided that $\ell \geq \delta(\mathbf{m})$.

\section{Local Properties of Extensions}

In this section, we study Cohen-Macaulayness of tangent cones of extensions of a toric variety having a Cohen-Macaulay tangent cone, see $[1,12,16]$ for the literature about Cohen-Macaulayness of tangent cones. We also show that if the local ring of a toric variety is of homogeneous type or has a non-decreasing Hilbert function, then its extensions share the same property. As a main result, we demonstrate that in the framework of extensions it is very easy to create infinitely many new families of arbitrary dimensional and embedding codimensional local rings having non-decreasing Hilbert functions supporting Rossi's conjecture. This is important, as the conjecture is known only for local rings with small (co)dimension:

- Cohen-Macaulay rings of dimension 1 and embedding codimension 2, [6],

- Some Gorenstein rings of dimension 1 and embedding codimension 3, [2],

- Complete intersection rings of embedding codimension 2, [13],

- Some local rings of dimension $1,[3,18]$,

where embedding codimension of a local ring is defined to be the difference between its embedding dimension and dimension. For instance, if $\mathbb{A}^{n}$ is the smallest affine space containing $V_{S}$, then embedding dimension of the local ring of $V_{S}$ is $n$. Its dimension 
coincides with the dimension of $V_{S}$ and its embedding codimension is nothing but the codimension of $V_{S}$, i.e. $n-\operatorname{dim} V_{S}$.

Before going further, we need to recall some terminology and fundamental results which will be used subsequently. If $V_{S} \subset \mathbb{A}^{n}$ is a toric variety, its associated graded ring is isomorphic to $K\left[x_{1}, \ldots, x_{n}\right] / I_{S}{ }^{*}$, where $I_{S}{ }^{*}$ is the ideal of the tangent cone of $V_{S}$ at the origin, that is the ideal generated by the polynomials $f^{*}$ with $f \in I_{S}$ and $f^{*}$ being the homogeneous summand of $f$ of the smallest degree. Thus, the tangent cone is CohenMacaulay if this quotient ring is also. Similarly, we can study the Hilbert function of the local ring associated to $V_{S}$ by means of this quotient ring, since the Hilbert function of the local ring is by definition the Hilbert function of the associated graded ring. Finally, we can find a minimal generating set for $I_{S}{ }^{*}$ by computing a minimal standard basis of $I_{S}$ with respect to a local order. For further inquiries and notations to be used, we refer to $[8]$.

Assume now that $V_{S_{\ell, \mathbf{m}}} \subset A^{n+1}$ is an extension of $V_{S}$, for suitable $\ell$ and $\mathbf{m}$. Then, by Proposition 2.4, we know that $I_{S_{\ell, \mathbf{m}}}=I_{S}+\langle F\rangle$, where $F=x_{n+1}^{\ell}-x_{1}^{s_{1}} \cdots x_{n}^{s_{n}}$.

Proposition 3.1. If $\mathcal{G}$ is a minimal standard basis of $I_{S}$ with respect to a negative degree reverse lexicographic ordering $\succ$ and $\ell \leq \Delta(\mathbf{m})$, then $\mathcal{G} \cup\{F\}$ is a minimal standard basis of $I_{S_{\ell, \mathbf{m}}}$ with respect to a negative degree reverse lexicographic ordering refining $\succ$ and making $x_{n+1}$ the biggest variable.

Proof. Let $\mathcal{G}^{\prime}=\mathcal{G} \cup\{F\}$. Since $N F(\operatorname{spoly}(f, g) \mid \mathcal{G})=0$, for all $f, g \in \mathcal{G}$, we have $N F\left(\operatorname{spoly}(f, g) \mid \mathcal{G}^{\prime}\right)=0$. Since $\operatorname{LM}(f) \in K\left[x_{1}, \ldots, x_{n}\right]$ and $\operatorname{LM}(F)=x_{n+1}^{\ell}$, it follows at once that $\operatorname{gcd}(\operatorname{LM}(f), \operatorname{LM}(F))=1$, for every $f \in \mathcal{G}$. Thus, we get $N F\left(\operatorname{spoly}(f, F) \mid \mathcal{G}^{\prime}\right)=0$, for any $f \in \mathcal{G}$. This reveals that $\mathcal{G}^{\prime}$ is a standard basis with respect to the afore mentioned local ordering and it is minimal because of the minimality of $\mathcal{G}$.

Theorem 3.2. If $V_{S} \subset A^{n}$ has a Cohen-Macaulay (Gorenstein) tangent cone at 0, then so have its extensions $V_{S_{\ell, \mathbf{m}}} \subset A^{n+1}$, provided that $\ell \leq \Delta(\mathbf{m})$.

Proof. An immediate consequence of the previous result is that $I_{S_{\ell, \mathbf{m}}}{ }^{*}=I_{S}{ }^{*}+\left\langle F^{*}\right\rangle$, where $F^{*}$ is $x_{n+1}^{\ell}$ whenever $\ell<\Delta(\mathbf{m})$ and is $F$ if $\ell=\Delta(\mathbf{m})$. In any case $F^{*}$ is a nonzerodivisor on $K\left[x_{1}, \ldots, x_{n+1}\right] / I_{S}{ }^{*}$ and thus $K\left[x_{1}, \ldots, x_{n+1}\right] / I_{S_{\ell, \mathbf{m}}}{ }^{*}$ is Cohen-Macaulay as required. In particular, both tangent cones have the same Cohen-Macaulay type.

Remark 3.3. Theorem 3.2 generalizes the results appeared in [1, Proposition 4.1] and [2, Theorem 3.6] from toric curves to toric varieties of any dimension. Moreover, Hilbert functions of the local rings of these extensions are nondecreasing in this case supporting Rossi's conjecture.

According to [9], a local ring is of homogeneous type if its Betti numbers coincide with the Betti numbers of its associated graded ring, considered as a module over itself. It is interesting to obtain local rings of homogeneous type, since in this case, for example, the local ring and its associated ring will have the same depth and their Cohen-Macaulayness will be equivalent since they always have the same dimension. It will also be easier to get information about the depth of the symmetric algebra in this case, see $[9,15]$. 
Proposition 3.4. If the local ring of $V_{S} \subset A^{n}$ is of homogeneous type, then its extensions will also have local rings of homogeneous type if and only if $\ell \leq \Delta(\mathbf{m})$.

Proof. Let $K[[S]]$ denote the local ring of $V_{S}$, i.e. the localization of the semigroup ring $K[S]=R / I_{S}$ at the origin, where $R=K\left[x_{1}, \ldots, x_{n}\right]$. The Betti numbers of $K[[S]]$ and $K[S]$ is the same, since localization is flat. For the convenience of notation let us use $G R[S]$ for the associated graded ring corresponding to $V_{S}$ and $\beta_{i}(G R[S])$ for the Betti numbers of the minimal free resolution of $G R[S]=R / I_{S}{ }^{*}$ over $R$.

Assume now that $K[[S]]$ is of homogeneous type, i.e. $\beta_{i}(K[[S]])=\beta_{i}(G R[S])$, for all $i$. For any extension $V_{S_{\ell, \mathbf{m}}} \subset A^{n+1}$ of $V_{S}$, we have from Proposition 2.4 that $I_{S_{\ell, \mathrm{m}}}=I_{S}+\langle F\rangle$, where $F=x_{n+1}^{\ell}-x_{1}^{s_{1}} \cdots x_{n}^{s_{n}}$. Therefore, by Remark 2.3, the Betti numbers are as follows

- $\beta_{1}\left(K\left[\left[S_{\ell, \mathbf{m}}\right]\right]\right)=\beta_{1}(K[[S]])+1$

- $\beta_{i}\left(K\left[\left[S_{\ell, \mathbf{m}}\right]\right]\right)=\beta_{i}(K[[S]])+\beta_{i-1}(K[[S]]), 2 \leq i \leq d=p d(K[[S]])$

- $\beta_{d+1}\left(K\left[\left[S_{\ell, \mathbf{m}}\right]\right]\right)=\beta_{d}(K[[S]])$.

If furthermore $\ell \leq \Delta(\mathbf{m})$, Proposition 3.1 yields $I_{S_{\ell, \mathbf{m}}}{ }^{*}=I_{S}{ }^{*}+\left\langle F^{*}\right\rangle$. Hence, by Remark 2.3, Betti numbers of $G R\left[S_{\ell, \mathbf{m}}\right]$ are found as:

- $\beta_{1}\left(G R\left[S_{\ell, \mathbf{m}}\right]\right)=\beta_{1}(G R[S])+1$

- $\beta_{i}\left(G R\left[S_{\ell, \mathbf{m}}\right]\right)=\beta_{i}(G R[S])+\beta_{i-1}(G R[S]), 2 \leq i \leq d=p d(K[[S]])$

- $\beta_{d+1}\left(G R\left[S_{\ell, \mathbf{m}}\right]\right)=\beta_{d}(G R[S])$.

It is obvious now that $\beta_{i}\left(G R\left[S_{\ell, \mathbf{m}}\right]\right)=\beta_{i}\left(K\left[\left[S_{\ell, \mathbf{m}}\right]\right]\right)$ for any $i$ and that local rings of extensions are of homogeneous type.

The converse is rather trivial, since homogeneity of local rings of extensions force that $\beta_{1}\left(G R\left[S_{\ell, \mathbf{m}}\right]\right)=\beta_{1}\left(K\left[\left[S_{\ell, \mathbf{m}}\right]\right]\right)$, i.e. $I_{S_{\ell, \mathbf{m}}}{ }^{*}=I_{S}{ }^{*}+\left\langle F^{*}\right\rangle$ which is possible only if $\ell \leq \Delta(\mathbf{m})$.

Finally, inspired by [3, Theorem 3.1], we consider extensions of a toric variety whose local ring has a non-decreasing Hilbert function and whose tangent cone is not necessarily Cohen-Macaulay. The proof is a modification of that of [3, Theorem 3.1] and the reason for this is that there are toric surfaces having non-decreasing Hilbert functions but having Hilbert series expressed as a ratio of a polynomial with some negative coefficients. The Hilbert series of the toric variety in Example 3.6 item (3) is such an example:

$$
\left(1+3 t+6 t^{2}+8 t^{3}+9 t^{4}+7 t^{5}+3 t^{6}-t^{8}\right) /(1-t)^{2} .
$$

Theorem 3.5. If $V_{S} \subset A^{n}$ has a local ring with non-decreasing Hilbert function, then so have its extensions $V_{S_{\ell, \mathbf{m}}} \subset A^{n+1}$, provided that $\ell \leq \Delta(\mathbf{m})$. 
Proof. Let $R=K\left[x_{1}, \ldots, x_{n}\right]$. If $I$ is a graded ideal of $R$, then it is a standard fact that the Hilbert function of $R / I$ is just the Hilbert function of $R / \operatorname{LM}(I)$, where $\operatorname{LM}(I)$ is a monomial ideal consisting of the leading monomials of polynomials in $I$. Now, Proposition 3.1 reveals that $I_{S_{\ell, \mathrm{m}}}{ }^{*}=I_{S}{ }^{*}+\left\langle F^{*}\right\rangle$, where $F=x_{n+1}^{\ell}-x_{1}^{s_{1}} \cdots x_{n}^{s_{n}}$ and that $\operatorname{LM}\left(I_{S_{\ell, \mathrm{m}}}{ }^{*}\right)=$ $\operatorname{LM}\left(I_{S}{ }^{*}\right)+\left\langle\operatorname{LM}\left(F^{*}\right)\right\rangle$. Since $\operatorname{LM}\left(I_{S}{ }^{*}\right) \subset R$ and $\operatorname{LM}\left(F^{*}\right)=x_{n+1}^{\ell}$ with respect to the local order mentioned in Proposition 3.1, it follows from the proof of [5, Proposition 2.4] that $R^{\prime}=R_{1} \otimes_{K} R_{2}$, where

$$
R^{\prime}=R\left[x_{n+1}\right] / \operatorname{LM}\left(I_{S_{\ell, \mathbf{m}}}{ }^{*}\right), R_{1}=R / \operatorname{LM}\left(I_{S}{ }^{*}\right) \text { and } R_{2}=K\left[x_{n+1}\right] /\left\langle x_{n+1}^{\ell}\right\rangle .
$$

Hilbert series of $R_{1}$ can be given as $\sum_{k \geq 0} a_{k} t^{k}$, where $a_{k} \leq a_{k+1}$ for any $k \geq 0$, since from the assumption the local ring associated to $V_{S}$ has non-decreasing Hilbert function. It is clear that the Hilbert series of $R_{2}$ is $h_{2}(t)=1+t+\cdots+t^{\ell-1}$. Since the Hilbert series of $R^{\prime}$ is the product of those of $R_{1}$ and $R_{2}$, we observe that the Hilbert series of $R^{\prime}$ is given by

$$
\begin{aligned}
\sum_{k \geq 0} b_{k} t^{k} & =\left(1+t+\cdots+t^{\ell-1}\right) \sum_{k \geq 0} a_{k} t^{k} \\
& =\sum_{k \geq 0} a_{k} t^{k}+\sum_{k \geq 0} a_{k} t^{k+1}+\cdots+\sum_{k \geq 0} a_{k} t^{k+\ell-1} \\
& =\sum_{k \geq 0} a_{k} t^{k}+\sum_{k \geq 1} a_{k-1} t^{k}+\cdots+\sum_{k \geq \ell-1} a_{k-\ell+1} t^{k} .
\end{aligned}
$$

Therefore, the Hilbert series $\sum_{k \geq 0} b_{k} t^{k}$ of $R^{\prime}$ is given by

$$
a_{0}+\left(a_{0}+a_{1}\right) t+\cdots+\left(a_{0}+\cdots+a_{\ell-2}\right) t^{\ell-2}+\sum_{k \geq \ell-1}\left(a_{k}+a_{k-1}+\cdots+a_{k-\ell+1}\right) t^{k} .
$$

It is now clear that $b_{k} \leq b_{k+1}$, for any $0 \leq k \leq \ell-2$, from the first part of the last equality above, since $a_{k} \leq a_{k+1}$. For all the other values of $k$, i.e. $k \geq \ell-1$, we have $b_{k}-b_{k+1}=a_{k-\ell+1}-a_{k+1} \leq 0$ which accomplishes the proof.

Example 3.6. In the following, we will say that the extension is nice if $\ell \leq \Delta(\mathbf{m})$.

1. The local ring of the affine cone of a projective toric variety is always of homogeneous type, for instance, $S=\{(3,0),(2,1),(1,2),(0,3)\}$ defines a projective toric curve in $\mathbb{P}^{3}$ and its affine cone is the toric surface $V_{S} \subset \mathbb{A}^{4}$ with the homogeneous toric ideal $I_{S}=\left\langle x_{2}^{2}-x_{1} x_{3}, x_{3}^{2}-x_{2} x_{4}, x_{2} x_{3}-x_{1} x_{4}\right\rangle$. Thus by Proposition 3.4, its affine nice extensions will have homogeneous type local rings which are not necessarily homogeneous. Take for example, $\ell=1$ and $\mathbf{m}=(0,3 s)$ for any $s>1$. Then, although $I_{S_{\ell, \mathrm{m}}}=I_{S}+\left\langle x_{4}^{s}-x_{5}\right\rangle$ is not homogeneous, its local ring is of homogeneous type.

2. Similarly, one can produce Cohen-Macaulay tangent cones using arithmetically Cohen-Macaulay projective toric varieties, since the toric ideal $I_{S}$ of their affine 
cones are homogeneous and thus $I_{S}=I_{S}{ }^{*}$. Therefore, all of their affine nice extensions will have Cohen-Macaulay tangent cones and local rings with non-decreasing Hilbert functions, by Theorem 3.2. The toric variety $V_{S} \subset \mathbb{A}^{4}$ considered in the previous item (1) and its nice extensions illustrate this as well.

3. Take $S=\{(6,0),(0,2),(7,0),(6,4),(15,0)\}$. Then it is easy to see that $I_{S}=$ $\left\langle x_{1} x_{2}^{2}-x_{4}, x_{3}^{3}-x_{1} x_{5}, x_{1}^{5}-x_{5}^{2}\right\rangle$. Since $V_{S} \subset \mathbb{A}^{5}$ is a toric surface of codimension $3, I_{S}$ is a complete intersection and thus the local ring of $V_{S}$ is Gorenstein. But, the tangent cone at the origin, is determined by $I_{S}{ }^{*}=\left\langle x_{5}^{2}, x_{4}, x_{3}^{3} x_{5}, x_{3}^{6}, x_{1} x_{5}\right\rangle$ and thus is not Cohen-Macaulay. Nevertheless, its Hilbert function $H_{S}$ is non-decreasing: $H_{S}(0)=1, H_{S}(1)=4, H_{S}(2)=8, H_{S}(3)=13, H_{S}(r)=6 r-6$, for $r \geq 4$. Consider now all nice extensions of $V_{S}$; defined by the following semigroups $S_{\ell, \mathbf{m}}=$ $\{(6 \ell, 0),(0,2 \ell),(7 \ell, 0),(6 \ell, 4 \ell),(15 \ell, 0), \mathbf{m}\}$. Therefore, Theorem 3.5 produces infinitely many new toric surfaces with local rings of dimension 2 and embedding codimension 4 whose Hilbert functions are non-decreasing even though their tangent cones are not Cohen-Macaulay. Indeed, one may produce this sort of examples in any embedding codimension by taking a sequence of nice extensions of the same example, since in each step the embedding codimension increases by one.

\section{References}

[1] F. Arslan, Cohen-Macaulayness of tangent cones, Proc. Amer. Math. Soc. 128 (2000), 2243-2251.

[2] F. Arslan and P. Mete, Hilbert functions of Gorenstein monomial curves, Proc. Amer. Math. Soc. 135 (2007), 1993-2002.

[3] F. Arslan, P. Mete and M. Şahin, Gluing and Hilbert functions of monomial curves, Proc. Amer. Math. Soc. 137 (2009), 2225-2232.

[4] D. Bayer and M. Stillman, Macaulay, A system for computations in algebraic geometry and commutative algebra, 1992, available at www.math.columbia.edu/ bayer/Macaulay.

[5] D. Bayer and M. Stillman, Computation of Hilbert functions, J. Symbolic Comput. 14 (1992), 31-50.

[6] J. Elias, The Conjecture of Sally on the Hilbert Function for Curve Singularities, J. Algebra 160 (1993), 42-49.

[7] P. A. Garcia-Sanchez and I. Ojeda, Uniquely presented finitely generated commutative monoids, Pacific J. Math. 248 (2010), 91-105.

[8] G.-M. Greuel, G. Pfister, A Singular Introduction to Commutative Algebra, SpringerVerlag, 2002.

[9] J. Herzog, M. E. Rossi and G. Valla, On the depth of the symmetric algebra, Trans. Amer. Math. Soc. 296 (1986), 577-606.

[10] M. Morales, Noetherian symbolic blow-ups, J. Algebra 140 (1991), 12-25. 
[11] M. Morales and A. Thoma, Complete intersection lattice ideals, J. Algebra 284 (2005), 755-770.

[12] D. P. Patil and G. Tamone, On the Cohen-Macaulayness of some graded rings, J. Algebra Appl. 7 (2008), 109-128.

[13] T. J. Puthenpurakal, The Hilbert function of a maximal Cohen-Macaulay module, Math. Z. 251 (2005), 551-573.

[14] J.C. Rosales, On presentations of subsemigroups of $\mathbb{N}^{n}$, Semigroup Forum 55 (1997), 152-159.

[15] M. E. Rossi and L. Sharifan, Minimal free resolution of a finitely generated module over a regular local ring, J. Algebra 322 (2009), 3693-3712.

[16] T. Shibuta, Cohen-Macaulayness of almost complete intersection tangent cones, J. Algebra 319 (2008), 3222-3243.

[17] M. Şahin, Producing set-theoretic complete intersection monomial curves in $\mathbb{P}^{n}$, Proc. Amer. Math. Soc. 137 (2009), 1223-1233.

[18] G. Tamone, On the Hilbert function of some non-Cohen-Macaulay graded rings, Comm. Algebra 26 (1998), 4221-4231.

[19] A. Thoma, Affine semigroup rings and monomial varieties, Comm. Algebra 24 (1996), 2463-2471. 